\title{
Robust, fast and sensitive near-infrared continuous-filtering Vernier spectrometer
}

\author{
Chuang Lu, ${ }^{1}$ Francisco Senna Vieira, ${ }^{1,2}$ Aleksander \\ GŁUSZEK, ${ }^{3}$ iD ISAK SIlANDER, ${ }^{1}$ GRZEGORZ SOBOŃ, ${ }^{3}$ iD AND \\ Aleksandra Foltynowicz ${ }^{1,{ }^{*}}$ \\ ${ }^{1}$ Department of Physics, Umeå University, 90187 Umeå, Sweden \\ ${ }^{2}$ currently at VTT Technical Research Centre of Finland Ltd, Tekniikantie 1, 02150 Espoo, Finland \\ ${ }^{3}$ Laser \& Fiber Electronics Group, Faculty of Electronics, Wroctaw University of Science and Technology, \\ Wybrzeze Wyspianskiego 27, 50-370 Wroctaw, Poland \\ *aleksandra.foltynowicz@umu.se
}

\begin{abstract}
We present a new design of a robust cavity-enhanced frequency comb-based spectrometer operating under the continuous-filtering Vernier principle. The spectrometer is based on a compact femtosecond Er-doped fiber laser, a medium finesse cavity, a diffraction grating, a custom-made moving aperture, and two photodetectors. The new design removes the requirement for high-bandwidth active stabilization present in the previous implementations of the technique, and allows scan rates up to $100 \mathrm{~Hz}$. We demonstrate the spectrometer performance over a wide spectral range by detecting $\mathrm{CO}_{2}$ around $1575 \mathrm{~nm}(1.7 \mathrm{THz}$ bandwidth and $6 \mathrm{GHz}$ resolution) and $\mathrm{CH}_{4}$ around $1650 \mathrm{~nm}(2.7 \mathrm{THz}$ bandwidth and $13 \mathrm{GHz}$ resolution). We achieve absorption sensitivity of $5 \times 10^{-9} \mathrm{~cm}^{-1} \mathrm{~Hz}^{-1 / 2}$ at $1575 \mathrm{~nm}$, and $1 \times 10^{-7} \mathrm{~cm}^{-1} \mathrm{~Hz}^{-1 / 2} \mathrm{~cm}^{-1}$ at $1650 \mathrm{~nm}$. We discuss the influence of the scanning speed above the adiabatic limit on the amplitude of the absorption signal.
\end{abstract}

(C) 2021 Optical Society of America under the terms of the OSA Open Access Publishing Agreement

\section{Introduction}

Cavity-enhanced laser spectroscopy allows sensitive and selective in-situ detection of molecular species in the gas phase. Techniques based on continuous wave (CW) lasers, such as cavity ringdown spectroscopy [1,2], integrated cavity output spectroscopy [3,4], and optical feedback cavity-enhanced absorption spectroscopy [5,6], have already found applications in areas ranging from atmospheric and environmental sensing [7] to breath analysis [8]. However, their spectral coverage is limited by the CW laser sources. Cavity-enhanced spectrometers based on frequency combs provide much wider spectral bandwidth and the possibility to detect many species simultaneously with reduced influence of spectral interferences [9]. Their use has been restricted mostly to laboratory environments because of the relative complexity of the experimental setups. A notable exception is the field-deployed instrument for detection of $\mathrm{IO}, \mathrm{BrO}, \mathrm{NO}_{2}$ and $\mathrm{H}_{2} \mathrm{CO}$ in the UV range based on a frequency-doubled femtosecond Ti:Sapphire laser and a low-resolution grating spectrograph $[10,11]$.

Cavity-enhanced comb-based spectrometers employ detection schemes with various levels of complexity and performance. For operation outside the laboratory, the spectrometer design should be robust, preferably without high-bandwidth comb-cavity stabilization schemes, and it should allow utilizing the spectral range of the comb with resolution sufficient for detection of pressure-broadened lines at atmospheric conditions. Cavity-enhanced comb-based Fourier transform spectroscopy, using either the dual-comb approach [12] or Michelson interferometers $[9,13,14]$, offers high absorption sensitivity and spectral resolution limited by the comb mode width provided that the comb is kept in resonance with the cavity using a tight lock [15-18]. The simultaneously transmitted bandwidth is ultimately limited by cavity dispersion. Spectrometers based on virtually imaged phased arrays (VIPAs) [19-21] do not require tight comb-cavity 
locking; instead, the comb lines are dithered with respect to the cavity modes, and slow feedback is used to keep them close to the resonance condition. The transmitted dispersed spectrum is recorded using a camera, which limits the simultaneously acquired bandwidth, and retrieving the absorption spectra from the camera images requires a rather complex data analysis. Another approach to cavity-enhanced comb-based spectroscopy utilizes the Vernier filtering of the comb, in which a controlled mismatch between the cavity free spectral range (FSR) and the comb repetition rate $\left(f_{\text {rep }}\right)$ is introduced to transmit individual comb lines, or groups of comb lines $[22,23]$. The spectra are acquired by scanning the filtered comb lines across the comb bandwidth via tuning either the $F S R$ or $f_{\text {rep. }}$. The Vernier spectrometers that resolve individual comb lines [22] are suitable for precision spectroscopy and retrieval of transition frequencies and linewidths [24-26]. They operate with CCD cameras that limit the simultaneous bandwidth that can be recorded in one $f_{\text {rep }} / F S R$ scan. Moreover, unless the comb source and the cavity are well stabilized, they suffer from a rather poor detection sensitivity [22]. An approach that provides better absorption sensitivity is the so-called continuous-filtering Vernier spectroscopy (CF-VS), in which groups of comb lines, called Vernier orders (VOs), are transmitted through the cavity. This technique is immune to laser frequency-to-amplitude noise conversion, since the noise contributions from the different comb lines within one VO cancel [23]. After the cavity, the consecutive VOs are separated by a diffraction grating, and one of them is imaged on a single detector, eliminating the need for a CCD camera. In previous implementations of CF-VS [23,27-29], the spectral acquisition was done by mounting the grating on a galvo scanner rotating synchronously with the $f_{\text {rep }} / F S R$ scan. Because of the different responses of the galvo scanner and the actuators controlling $f_{\text {rep }} / F S R$, this synchronization was actively controlled to ensure a fixed direction of the beam propagation after the grating. An error signal was obtained using a position sensing detector, and a relatively fast (10-100 kHz bandwidth) feedback to the laser cavity length and/or the enhancement cavity length was used to synchronize the two scans. The feedback gain depended on the transmitted power, limiting the spectral range that could be scanned and leading to distortions in strong absorption lines [28]. Moreover, the load on the galvo scanner limited the scan rate to about $20 \mathrm{~Hz}[28,29]$.

Here we present a new, improved approach to CF-VS based on a compact, free-running Er-doped fiber laser, a fixed diffraction grating, a rotating chopper wheel with a custom-made blade that acts as a moving aperture for selecting the VO, and imaging lenses in front of the detectors. We show that the system can operate in a free-running mode. For long-term stability, we implement a simple low-bandwidth stabilization scheme that does not require a position sensing detector - instead, it uses an error signal derived from the detector that measures the spectrum at the frequency of the scan already present in the system. The correction signal is sent to the enhancement cavity length, i.e., no feedback to the laser is needed, which makes the spectrometer compatible with other comb sources operating in the same wavelength range. We demonstrate the performance of the spectrometer by detecting $\mathrm{CO}_{2}$ and $\mathrm{CH}_{4}$ in two different spectral ranges. We investigate the influence of the scan rate on the signal strength, and we develop a simple model that allows retrieving the gas concentration at non-adiabatic scanning conditions.

\section{Scanning speed dependence}

When the cavity length is set to match the free spectral range (FSR) to the $m^{\text {th }}$ multiple of the laser repetition rate $\left(f_{\text {rep }}\right)$ and the laser carrier-envelope offset frequency is set equal to the cavity offset frequency, every $m^{\text {th }}$ comb line is transmitted through the cavity (within the range determined by the cavity dispersion). The corresponding cavity length is called the perfect match length $\left(L_{\mathrm{PM}}\right)$ and it is given by $c /\left(2 m f_{\text {rep }}\right)$, where $c$ is the speed of light. In CF-VS [27], the cavity length is detuned from $L_{\mathrm{PM}}$ by $\Delta L \ll L_{\mathrm{PM}} / F$, where $F$ is the cavity finesse. This introduces a mismatch between the $F S R$ and $m f_{\text {rep }}$, i.e., an $F S R / m f_{\text {rep }}$ ratio of $L_{\mathrm{PM}} /\left(L_{\mathrm{PM}}+\Delta L\right)$. As a result, 
groups of comb lines, i.e. the Vernier orders, separated in the optical domain by $c /(2 m|\Delta L|)$, are transmitted through the cavity. The FWHM of the Vernier order, given by $\Gamma_{\mathrm{v}}=c /(2|\Delta L| F)$, determines the resolution of the spectrometer. The center frequency of the $k^{\text {th }} \mathrm{VO}$, given by $v_{\mathrm{k}}=c\left(k-\delta f_{0} / f_{\text {rep }}\right) /(2 m|\Delta L|)$, where $\delta f_{0}$ is the difference between the offset frequencies of the comb and the cavity, can be swept across a spectral range by scanning the cavity length, or, alternatively, the $f_{\text {rep }}$. From this equation, the VO number at a given wavelength within the comb spectrum, $\lambda_{\mathrm{k}}$, is given by $k=2 m|\Delta L| / \lambda_{\mathrm{k}}+\delta f_{0} / f_{\text {rep }}$. Since the term $\delta f_{0} / f_{\text {rep }}$ is $\leq 1$, it introduces an uncertainty of one VO in the VO number determination based on the value of $\Delta L$ if $\delta f_{0}$ is not known.

In the presence of an intra-cavity absorber with frequency-dependent absorption $(\alpha)$ and dispersion $(\phi)$ coefficients, the cavity transmitted intensity $I^{\mathrm{T}}\left(v_{k}\right)$ can be expressed as $[27,28]$

$$
I^{\mathrm{T}}\left(v_{k}\right) \approx \int_{v_{k}-F S R_{V} / 2}^{v_{k}+F S R_{V} / 2} \frac{1-\alpha(v) L}{\left[1+\frac{F \alpha(v) L}{\pi}\right]^{2}} \times \frac{T_{\mathrm{res}}}{1+\left\{\frac{v-v_{k}-[c \phi(v) / 4 \pi](L / \Delta L)}{c /(4 F L L+[c \alpha(v) / 4 \pi] \mid L / \Delta L)}\right\}^{2}} \frac{I(v)}{m f_{\mathrm{rep}}} \mathrm{d} v
$$

where $T_{\text {res }}$ is the on-resonance cavity transmission, and $I(v)$ is the intensity profile of the comb. The above equation is valid only in the adiabatic limit, i.e., at spectral scanning speeds low enough so that the cavity mode transfer function is Lorentzian. The adiabatic regime of the spectral scanning is achieved when the duration of the passage of one comb line through the FWHM of the cavity resonance $\left[\Gamma_{\mathrm{c}}=c /\left(2 L_{\mathrm{PM}} F\right)\right]$ is much longer than the cavity ringdown time $\left(\tau_{\mathrm{RD}}\right)$, given by $1 /\left(2 \pi \Gamma_{\mathrm{c}}\right)[30]$. Therefore, the adiabatic scanning speed (in units of $\mathrm{Hz} / \mathrm{s}$ ) for a single-mode laser should be much lower than $\Gamma_{\mathrm{c}} / \tau_{\mathrm{RD}}=2 \pi \Gamma_{c}^{2}$. In CF-VS, tuning the cavity modes (or the comb lines) over a given spectral range corresponds to tuning of the VO center frequency magnified by a factor of $L_{\mathrm{PM}} / \Delta L$ [27]. Therefore, we define a cut-off scanning speed ( $W_{\text {cut-off }}$ ) for a $\mathrm{VO}$ as

$$
W_{\text {cut - off }}=2 \pi \Gamma_{c}^{2} \frac{L_{\mathrm{PM}}}{\Delta L}=2 \pi \Gamma_{c} \Gamma_{v}=\frac{\pi c^{2}}{2 \Delta L L_{\mathrm{PM}} F^{2}} .
$$

Deriving an equation for transmitted intensity valid for scanning speeds above the adiabatic limit would require assuming the cavity mode profile modified by the cavity ringdown signal, which has not been done in the literature. Here, we introduce a scanning-speed-dependent Lorentzian correction function, $\mathrm{C}(W)$, with FWHM equal to $W_{\text {cut-off }}$, as a first order approximation of the influence of the non-adiabatic scanning speed. This Lorentzian correction function is given by

$$
\mathrm{C}(W)=\frac{C_{0}}{1+\left(\frac{2 W}{W_{\text {cut }- \text { off }}}\right)^{2}},
$$

and it multiplies the finesse in Eq. (1) to model the loss of the enhancement of the interaction length as the comb lines are tuned away from the cavity resonances before the intracavity field reaches the stationary state. In Eq. (3), $C_{0}$ is a constant scaling factor used to correct any inaccuracy in the cavity finesse determination. According to this equation, the cut-off scanning speed corresponds to a condition at which the enhancement of the signal drops to $20 \%$ of the initial (adiabatic) value. It is worth noting that the adiabatic scanning speed limit in Ref. [27] is defined as $W_{\text {cut-off }} /(2 \pi)$, where the enhancement is equal to $90 \%$ of the adiabatic value.

\section{Experimental setup and procedures}

The schematics of the CF-VS setup is shown in Fig. 1. The setup is based on a compact femtosecond Er-doped fiber laser, a medium finesse cavity, and a detection system consisting of a fixed diffraction grating, rotating wheel with a slit acting as a moving aperture, and two photodetectors. All components of the system and the acquisition procedures are described in the 


\section{Optics EXPRESS}

sections below. The 3D Visualization 1 in the supplementary material shows the spectrometer under operation.

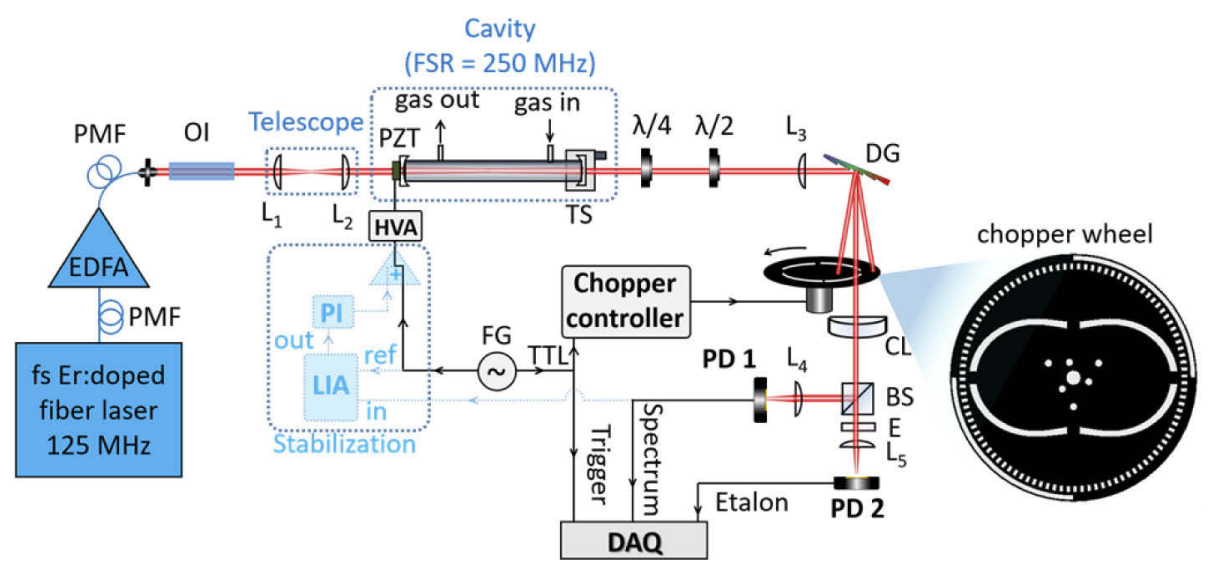

Fig. 1. Experimental setup. EDFA: Er-doped fiber amplifier; PMF: polarization maintaining fiber; OI: optical isolator; $\mathrm{L}_{1}-\mathrm{L}_{5}$ : spherical lenses; PZT: piezoelectric transducer; HVA: high voltage amplifier; TS: translation stage; $\lambda / 4$ : quarter-wave plate; $\lambda / 2$ : half-wave plate; DG: diffraction grating; CL: cylindrical lens; BS: non-polarizing beamsplitter; E: 3-mm-thick silicon etalon; $\mathrm{PD}_{1} \& \mathrm{PD}_{2}$ : InGaAs photodetectors; PI: proportional integral controller; LIA: lock-in amplifier; FG: function generator; DAQ: multichannel data acquisition card.

\subsection{Compact femtosecond Er-doped fiber laser source}

The spectrometer is based on an entirely fiberized polarization-maintaining (PM) femtosecond Er-doped fiber laser source, described in detail in Ref. [31]. The source consists of a thermally stabilized oscillator with $125 \mathrm{MHz}$ repetition rate and an amplifier. The low power $(5 \mathrm{~mW}, 335$ fs) pulses from the oscillator are amplified in the Er-doped fiber amplifier (EDFA) that boosts the average power to $250 \mathrm{~mW}$ and compresses the pulses to $45 \mathrm{fs}$. The amplifier output has FWHM bandwidth of $62 \mathrm{~nm}$ around $1560 \mathrm{~nm}$, which enables the measurement of the $3 v_{1}+v_{3}$ band of $\mathrm{CO}_{2}$. To further extend the spectral coverage of the source, the output of the amplifier is sent through a 4-m-long single-mode PM fiber (Nufern PM1550XP) of the same type as the fiber at the output of the amplifier, which shifts the spectrum to $1650 \mathrm{~nm}$ with $27 \mathrm{~nm}$ FWHM bandwidth via the soliton self-frequency shift effect. This output allows measuring spectra of the $2 v_{3}$ band of $\mathrm{CH}_{4}$. The laser is operated in a free-running mode, i.e., its $f_{\text {rep }}$ and $f_{0}$ are not stabilized.

\subsection{Cavity}

The output of the laser is directed to free space, passes an optical isolator, and is mode-matched to a cavity using a telescope (lenses $\mathrm{L}_{1} \& \mathrm{~L}_{2}$, both with $75 \mathrm{~mm}$ focal length). The cavity is 60 $\mathrm{cm}$ long and made of 2 mirrors with 5-m radius of curvature and maximum reflectivity at 1575 $\mathrm{nm}$. The wavelength dependence of the cavity finesse was characterized using cavity ringdown spectroscopy, and modelled by a $3^{\text {rd }}$ order polynomial fit to the experimental values. The mean finesse was found to be around 810 at $1578 \mathrm{~nm}$ and around 490 at $1650 \mathrm{~nm}$, and varied by up to $5 \%$ depending on the cavity alignment, which we attribute to imperfections (inhomogeneity) in the cavity mirror coatings. We note that the accuracy of the measurement at $1650 \mathrm{~nm}$ was limited by the short ringdown time.

The cavity FSR is $250 \mathrm{MHz}$, equal to twice the comb repetition rate, and therefore every other comb line is transmitted through the cavity at perfect match condition $(m=2)$. The mirrors are mounted in holders that are attached to a tube with an inlet and outlet for the gas sample. One 
holder is mounted on a translation stage to allow coarse tuning of the cavity length around $L_{\mathrm{PM}}$ to set the desired $F S R / f_{\text {rep }}$ ratio, and thus the spectral resolution. The second holder contains a piezoelectric transducer (PZT) that allows dithering the cavity length in order to scan the selected VO across the desired spectral region. The PZT is driven via a high voltage amplifier (HVA) by sinusoidal modulation from a low-noise function generator (FG).

\subsection{Detection system}

The beam transmitted through the cavity is incident onto a gold-coated reflective diffraction grating with line density of $900 \mathrm{l} / \mathrm{mm}$ (Spectrogon, PC 0900) that disperses the consecutive VOs. The grating is mounted on a rotational stage to guide the selected spectral region (around 1580 $\mathrm{nm}$ or $1650 \mathrm{~nm}$ ) into the spectrometer. A quarter- and a half-wave plate before the grating are used to control the polarization to maximize the diffraction efficiency and minimize the influence of the crosstalk between the light propagating along the two axes of the PM fiber [32]. After the grating, the different VO beams are incident on a custom-made wheel (see inset in Fig. 1) mounted on a commercial chopper driver (Thorlabs, MC2000B-EC) referenced to the TTL output of the function generator driving the cavity PZT. The VO beams are focused to a diameter of $0.6 \mathrm{~mm}$ at the position of the wheel using a $500 \mathrm{~mm}$ focal length lens $\left(\mathrm{L}_{3}\right)$ positioned after the cavity. A 4-mm-wide slit, narrower than the spatial separation between the consecutive VOs at the position of the chopper wheel, is cut in the wheel to transmit only one VO order. The pattern of the slit is designed to act as an aperture moving sinusoidally in the horizontal direction during the wheel rotation (see Visualization 1). The outer slots provide frequency tracking as feedback to the chopper driver. The cavity PZT scan and the wheel rotation are passively synchronized by adjusting the phase shift on the chopper driver and the amplitude of the FG signal driving the PZT. The range of the spectral scan is determined by the amplitude of the aperture movement along the horizontal direction and can be adjusted by tuning the horizontal angle of the wheel with respect to the beam.

After the chopper wheel, the beam is incident on a cylindrical lens (CL, $200 \mathrm{~mm}$ focal length) positioned $200 \mathrm{~mm}$ after the grating to ensure a parallel movement of the selected VO after the lens during the spectral scan. A non-polarizing beamsplitter then divides the beam. The transmitted beam is sent through a 3-mm-thick uncoated silicon window (Thorlabs, WG80503, $\mathrm{FSR} \approx 14 \mathrm{GHz})$ and focused using a spherical lens $\left(\mathrm{L}_{4}, 25.4 \mathrm{~mm}\right.$ diameter, $50 \mathrm{~mm}$ focal length) on an InGaAs photodetector ( $\mathrm{PD}_{2}$, Thorlabs, PDA20CS-EC) for frequency calibration. The reflected beam is focused using a similar lens $\left(\mathrm{L}_{5}\right)$ directly on a second photodetector of the same type ( $\mathrm{PD}_{1}$, Thorlabs, PDA20CS-EC) for the acquisition of the spectrum. The outputs of $\mathrm{PD}_{1}$ and $\mathrm{PD}_{2}$ are digitized using a multichannel data acquisition card (DAQ, National Instruments, PCI-6122) with a $500 \mathrm{kS} / \mathrm{s}$ sampling rate, which is triggered by the TTL output of the function generator.

\subsection{Optional low-bandwidth stabilization}

Since the slit size $(4 \mathrm{~mm})$ is larger than the beam diameter $(0.6 \mathrm{~mm})$ and smaller than the VO separation $(8 \mathrm{~mm})$, the passive synchronization has a certain degree of tolerance for the different responses of the chopper and the PZT at the scanning frequency. Therefore, the selected VO passes through the moving slit as long as the mean $F S R / f_{\text {rep }}$ ratio is relatively stable; the size of the slit allows a tolerance of $\sim 40 \mathrm{~Hz}$ change in $F S R$ which corresponds to a $\sim 200 \mathrm{~nm}$ deviation in the cavity length (or $\sim 20 \mathrm{~Hz}$ change in $f_{\text {rep }}$ ). Thus, unlike in previous demonstrations using a rotating grating, no active high-bandwidth feedback to the laser $f_{\text {rep }}$ or cavity length is required. To compensate for slow drifts of the cavity length and/or the laser $f_{\text {rep }}$, i.e., to keep the mean $F S R / f_{\text {rep }}$ ratio constant over a long time, active stabilization can be implemented using the modulation already present in the system. The components employed in the stabilization are depicted as light blue boxes in Fig. 1. The output of $\mathrm{PD}_{1}$ is demodulated using a lock-in amplifier 
(LIA, Stanford Research Systems, SR830) referenced to the sinusoidal signal from the function generator that drives the cavity PZT. The first harmonic output of the LIA is used as an error signal and sent to a proportional-integral (PI) controller (Newport LB1005) with a $10 \mathrm{~Hz}$ corner frequency. The slow correction signal is summed with the modulation signal from the FG and sent to the HVA that scans the cavity length. This centers the laser spectral envelope with respect to the scan of the aperture.

\subsection{Spectral acquisition procedures}

To demonstrate the performance of the spectrometer, the target gases, $\mathrm{CO}_{2}$ and $\mathrm{CH}_{4}$, are detected in two spectral regions. Prior to every measurement series, the perfect match length $\left(L_{\mathrm{PM}}=2 f_{\text {rep }}\right)$ is found by translating the cavity mirror to maximize the peak transmitted power while the other mirror is simultaneously modulated using the PZT. The cavity length is then detuned by $\Delta L=-30(2) \mu \mathrm{m}$ from $L_{\mathrm{PM}}$, where the uncertainty originates from the resolution of the translation stage. This setting corresponds to a $\mathrm{VO}=-76(5)$ at $1575 \mathrm{~nm}$, which yields a spectral resolution around $6 \mathrm{GHz}$ for detection of $\mathrm{CO}_{2}$, and $\mathrm{VO}=-73(5)$ at $1650 \mathrm{~nm}$, which yields a spectral resolution around $10 \mathrm{GHz}$ for detection of $\mathrm{CH}_{4}$. We neglect the influence of the $\delta f_{0} / f_{\text {rep }}$ term on the $\mathrm{VO}$ number determination, since it introduces an uncertainty below that from the resolution of the translation stage. The scan rate is set by adjusting the frequency of the output signal of the function generator. Afterward, the phase shift on the chopper driver and the amplitude setting of the function generator are adjusted to synchronize the rotation of the wheel and the scan of the cavity length. The synchronization ensures a minimum spatial variation of the selected VO with respect to the slit center during the spectral scan. The synchronization is confirmed by observing reproducible spectra staying at the center of the acquisition window. The PI controller for active stabilization can be turned on to prevent long-term drifts.

Once the spectral scan conditions are set, the cavity is filled with pure $\mathrm{N}_{2}$ for the acquisition of background spectra. Before the spectra of the target gases are measured, the cavity is flushed with the respective gas at a flow rate of $800 \mathrm{~mL} / \mathrm{min}$ until changes in the amplitude of the absorption lines are no longer visible. During the measurement of $\mathrm{CO}_{2}$, the gas flow is turned off and the cavity is closed before data acquisition. For the measurement of $\mathrm{CH}_{4}$, a flow of $100 \mathrm{~mL} / \mathrm{min}$ is maintained. All measurements are performed at atmospheric pressure and the available gas samples were $1 \% \mathrm{CO}_{2}$ and $45 \mathrm{ppm} \mathrm{CH}_{4}$ in $\mathrm{N}_{2}$.

The relative frequency axis is retrieved using the signal of the silicon etalon, as described in detail in Ref. [29]. The output of $\mathrm{PD}_{2}$ is normalized by the output of $\mathrm{PD}_{1}$ to remove the absorption signal, and bandpass filtered to remove the DC component and high-frequency noise. Next, the zero crossings of the silicon etalon fringes are used as frequency markers for resampling of the spectrum. The absolute frequency scale is calibrated by comparing the positions of absorption lines in the experimental spectrum with simulations based on the HITRAN 2016 database [33]. The values of the scanning speed (in units of THz/ms) at the zero crossings on the etalon fringes are determined by dividing half of the etalon FSR by the corresponding acquisition time. The full profile of the wavelength-dependent scanning speed is obtained by linearly interpolating these values on the frequency scale of the acquired spectrum.

\section{Results}

\subsection{Free-running versus stabilized spectrometer}

To evaluate the performance of the CF-VS spectrometer operating without and with the active stabilization of the $F S R / f_{\text {rep }}$ ratio, we acquired spectra of $1 \% \mathrm{CO}_{2}$ under both conditions using a scan rate of $20 \mathrm{~Hz}$, as shown in Figs. 2(a) and 2(b), respectively. Without the active lock (i.e., in the free-running mode), the center of the spectrum drifts slowly over time, but as long as it remains within the acquisition window (i.e., the same VO is being monitored) spectral 
information can be extracted from it without distortion. The slow active stabilization ensures that the spectrum is centered within the acquisition window over a long period of time, as shown in Fig. 2(b). We note that the spectrometer allows measuring the entire bandwidth of the comb source (FWHM of $62 \mathrm{~nm}$ ), and Fig. 2 shows a zoom of the range where $\mathrm{CO}_{2}$ absorbs.

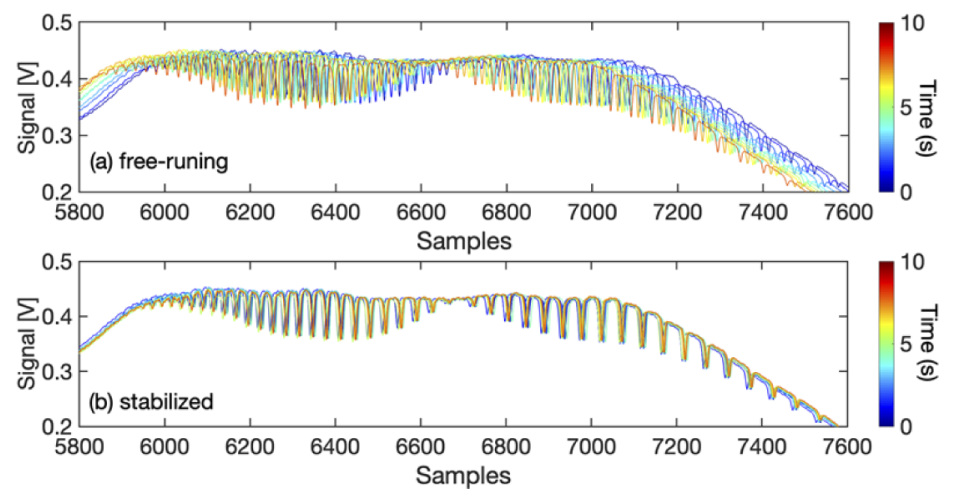

Fig. 2. Raw spectra of $1 \% \mathrm{CO}_{2}$ in $\mathrm{N}_{2}$ at atmospheric pressure from a 10 s series of 200 scans acquired at a scan rate of $20 \mathrm{~Hz}$ with the spectrometer operating (a) in the free-running mode and (b) with the active stabilization. For clarity, only every $20^{\text {th }}$ scan in the series is shown.

\section{2. $\mathrm{CO}_{2}$ detection at non-adiabatic scanning speeds}

To demonstrate the capability of the spectrometer for high-speed acquisition, we measured spectra of $1 \% \mathrm{CO}_{2}$ in $\mathrm{N}_{2}$ at scan rates from $10 \mathrm{~Hz}$ to $100 \mathrm{~Hz}$. As an example, Fig. 3 shows the background-normalized spectra (markers) of $\mathrm{CO}_{2}$ from single scans acquired at a scan rate of (a) $10 \mathrm{~Hz}$ and (b) $50 \mathrm{~Hz}$. The number of points per absorption line is lower in the spectrum recorded at the higher scan rate because the sampling rate of the DAQ card is equal for all spectra. Since the scan of the cavity PZT is sinusoidal, the instantaneous scanning speed changes during the scan, as shown in the top panels. The oscillations in the scanning speed originate from the uncertainty in the determination of the zero-crossings of the etalon fringes. The red curves in Fig. 3 show the fit of the modified model of the Vernier spectrum (detailed in section 2) to the $\mathrm{P}$ branch of the spectrum, where the scanning speed is relatively constant. The baseline remaining after the background normalization is corrected by fitting a sum of a $5^{\text {th }}$ order polynomial and sine waves together with the Vernier model. In the model, the $\mathrm{CO}_{2}$ line parameters are taken from the HITRAN 2016 database [33], and the pressure and temperature are set to 760 Torr and $295 \mathrm{~K}$, respectively. The VO number is fixed to -76, as expected from the cavity length setting. Because of the large uncertainty in the cavity finesse (see section 3.2), we fix the concentration of $\mathrm{CO}_{2}$ to $1 \%$, and use the correction function $(C)$ from Eq. (3) as the only free parameter in the spectral fitting routine.

The general agreement between the data and the model is good. The structure in the residuum for the spectrum acquired at $10 \mathrm{~Hz}$, shown in the lower panel of Fig. 3(a), is caused by the etalon fringes remaining after the baseline correction procedure and by uncertainties in the frequency scale calibration. Nevertheless, the relative amplitude of the residuum is lower than what was observed in previous CF-VS demonstrations employing the rotating diffraction grating and active stabilization using either an Er-doper fiber laser [31] or a mid-infrared optical parametric oscillator [28]. The improvement is brought partly by the lack of high-speed active stabilization whose gain depended on the amplitude of the signal (and thus distorted the absorption lines), and partly by the silicon etalon used for frequency calibration, which has a better fringe contrast than the $\mathrm{CaF}_{2}$ etalon used in the previous works. Altogether, this indicates that the quality of 


\section{Optics EXPRESS}

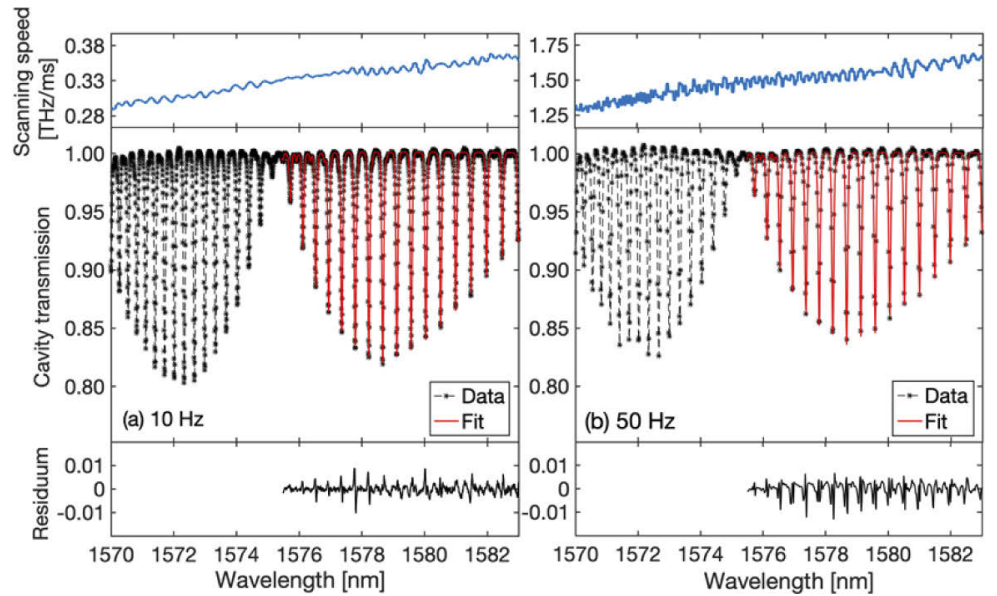

Fig. 3. Middle panels: spectra (markers) of $1 \% \mathrm{CO}_{2}$ acquired at a rate of (a) $10 \mathrm{~Hz}$ and (b) $50 \mathrm{~Hz}$ and the corresponding fits of the model (red curves). Top panels: the instantaneous scanning speeds vs wavelength. Bottom panels: residua of the fit.

the spectra recorded with the new type of spectrometer in the adiabatic limit is better than in previous demonstrations. The more pronounced residuum in the spectrum recorded at $50 \mathrm{~Hz}$ originates from a mismatch between the experimental data and the simple model at non-adiabatic scanning speed, as well as the reduced precision of the determination of the zero-crossings of the etalon caused by the lower relative sampling rate.

Figure 4 shows the correction factors $(C)$ obtained from fits to spectra recorded at different scan rates (blue markers) as a function of the scanning speed $(W)$, together with a fit (red curve) of Eq. (3) with cut-off speed ( $\left.W_{\text {cut-off }}\right)$ and the scaling factor $\left(C_{0}\right)$ as fitting parameters. The scanning speed was calculated as the mean of the instantaneous scanning speed in the fitting range $(\mathrm{P}$ branch), and the uncertainties are given by $2 / 3$ of the difference between the maximum and minimum scanning speeds within this range, averaged over 200 spectra. The correction factors are taken as the mean retrieved from 200 consecutive spectra, and the uncertainties are the standard deviations of these values. The bottom panel shows the residuum of the fit.

The fit yields a scaling factor $\left(C_{0}\right)$ of $0.94(1)$ and a cut-off speed ( $\left.W_{\text {cut-off }}\right)$ of $12.8(1.1) \mathrm{THz} / \mathrm{ms}$, where the uncertainties are the $95 \%$ confidence interval of the fit. The mean finesse in the $\mathrm{P}$ branch range, corrected by the scaling factor, is 760(20), where the uncertainty is the variation of the finesse across the fitting range. Using the mean value of the corrected finesse and Eq. (2), the expected cut-off speed is $13.2(1.1) \mathrm{THz} / \mathrm{ms}$. Here, the overall uncertainty is calculated as the in-quadrature sum of the uncertainties in the mean finesse and in the $\Delta L$ setting (see section 3.5). The $4 \%$ relative discrepancy between the expected and retrieved cut-off speeds is within the uncertainty margins of the two values, which validates the model.

\subsection{Sensitivity and long-term stability}

To evaluate the absorption sensitivity, we calculated the relative noise on the baseline $(\sigma)$ as the standard deviation of the ratio of two consecutive background spectra after baseline correction in the spectral region of the $\mathrm{CO}_{2}$ absorption band. The relative noise level was equal to 5.5(6) $\times 10^{-4}$ at scan rates up to $60 \mathrm{~Hz}$, because the sampling rate, and thus the electronic detection bandwidth, was constant for all spectra. This means that the noise equivalent absorption sensitivity (NEAS) is $5 \times 10^{-9} \mathrm{~cm}^{-1} \mathrm{~Hz}^{-1 / 2}$, calculated as $\sigma T^{1 / 2} / L_{\text {eff }}$, where $T$ is the acquisition time (16 ms at 60 $\mathrm{Hz}$ ) and $L_{\mathrm{eff}}=146 \mathrm{~m}$ is the effective path length, given by $F L_{\mathrm{PM}} / \pi$. The figure of merit, calculated 


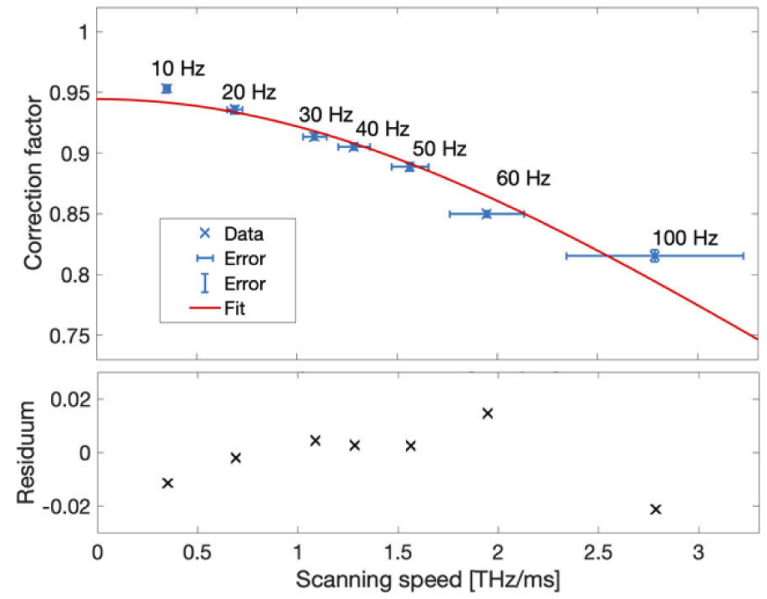

Fig. 4. Top: The correction function (blue markers) retrieved from fits to spectra recorded at different scanning speeds, together with the fit (red curve) of Eq. (3). Bottom: the residuum of the fit.

as NEAS $/ M^{1 / 2}$, is $4 \times 10^{-10} \mathrm{~cm}^{-1} \mathrm{~Hz}^{-1 / 2}$, where $M=136$ is the number of resolved elements $\left(\Gamma_{\mathrm{V}}=6.6 \mathrm{GHz}\right)$ in the spectral range used for the fit $(0.9 \mathrm{THz})$.

We performed the Allan-Werle analysis [34] to determine the $\mathrm{CO}_{2}$ concentration detection limit. For this, we recorded a 10 s series of spectra of $1 \% \mathrm{CO}_{2}$ in $\mathrm{N}_{2}$ and of pure $\mathrm{N}_{2}$ (background) at different scan rates using the actively stabilized spectrometer. The $\mathrm{CO}_{2}$ spectra were normalized to a mean background spectrum, and the concentration of $\mathrm{CO}_{2}$ was extracted from each spectrum using a fit of the Vernier model to the $\mathrm{P}$ branch with the correction function determined in the previous section. This time, the only fitting parameter was the $\mathrm{CO}_{2}$ concentration, whereas all other parameters and the baseline correction were the same as those described in section 4.2. We also fit the $\mathrm{CO}_{2}$ model to every $\mathrm{N}_{2}$ spectrum normalized to the same mean background spectrum used for normalization of the $\mathrm{CO}_{2}$ spectrum, and retrieved the concentration. Figure 5 shows the Allan-Werle plot of the $\mathrm{CO}_{2}$ concentration (in ppm) obtained from fits to the $1 \% \mathrm{CO}_{2}$ spectra (solid markers) and to the $\mathrm{N}_{2}$ spectra (open markers) at three different scan rates. The data follow the white noise behavior in both cases, as shown by the dashed black lines with slopes of $7.1 \mathrm{ppm}$ $\mathrm{Hz}^{-1 / 2}$ and $1.4 \mathrm{ppm} \mathrm{Hz}^{-1 / 2}$, respectively. The noise from the fits to $\mathrm{CO}_{2}$ spectra is about five times higher than for the $\mathrm{N}_{2}$ spectra, which can be attributed to the uncertainty in the frequency scale to which the background $\mathrm{N}_{2}$ spectra are less sensitive. The concentration detection limit is the same for all scan rates because the sampling rate was constant for all spectra. This implies that although the noise level is constant in single-scan spectra at different scan rates (as explained in the beginning of this section), the number of points per absorption line decreases linearly with the scan rate. This, in turn, implies that for a given acquisition time, the averaging effect is offset by the decrease of the number of points used for fitting [35].

\section{4. $\mathrm{CH}_{4}$ detection}

For detection of $\mathrm{CH}_{4}$, the spectrometer was tuned to $1650 \mathrm{~nm}$ by adding the longer PM fiber after the laser amplifier and rotating the diffraction grating. Figure 6 shows a single-scan background-corrected spectrum of $45 \mathrm{ppm} \mathrm{CH}_{4}$ in $\mathrm{N}_{2}$ at 760 Torr (black markers) recorded using a scan rate of $20 \mathrm{~Hz}$, compared to a fit of the Vernier model (red curve). The mean scanning speed extracted from the etalon signal is $0.45(5) \mathrm{THz} / \mathrm{ms}$, which is much slower than the expected cut-off speed ( $W_{\text {cut-off }}$ ) of $33 \mathrm{THz} / \mathrm{ms}$, calculated for a finesse of 490 . Therefore, in the fit to the spectrum, we fix the concentration of $\mathrm{CH}_{4}$ to $45 \mathrm{ppm}$, assume $C=C_{0}$ in Eq. (3), and use 


\section{Optics EXPRESS}

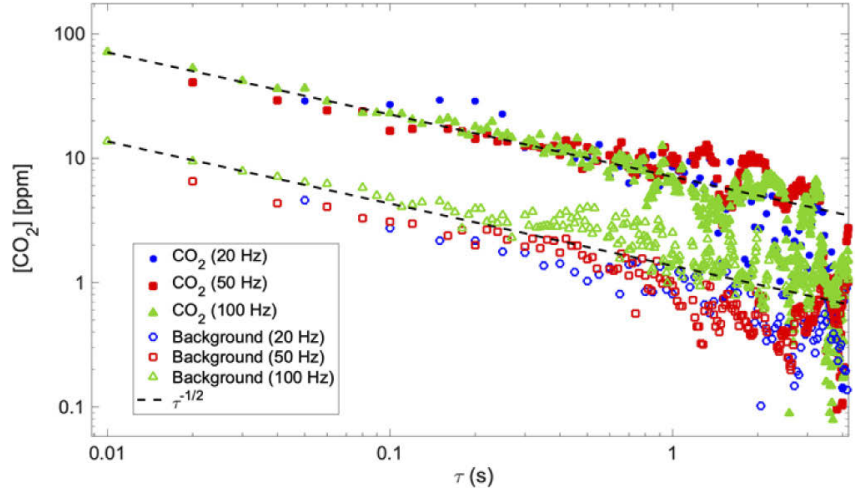

Fig. 5. The Allan-Werle plot for $\mathrm{CO}_{2}$ concentration retrieved from fits to $10 \mathrm{~s}$ measurement series of $\mathrm{CO}_{2}$ spectra (solid markers) and background spectra (open markers) at different scan rates. The dashed lines show the corresponding white noise regime in both cases.

$C_{0}$ as the only fitting parameter. The retrieved scaling factor is $0.75(1)$, with the uncertainty being the standard deviation of values from 200 consecutive fits. This implies that the finesse is $369(4)$, and the cut-off speed is 56(1) THz/ms (where the uncertainties are propagated from the scaling factor). Since the scanning speed is well below the cut-off speed, the unmodified Eq. (1) is valid, which is supported by the good agreement between the model and the data in Fig. 6. The structure of the residuum originates mostly from the spectral fringes that are not completely removed by the spectral normalization and baseline correction procedure.

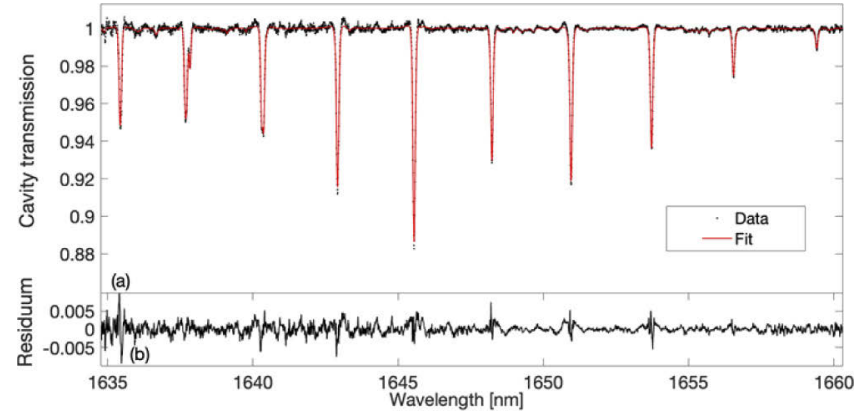

Fig. 6. Spectrum of $45 \mathrm{ppm}$ of $\mathrm{CH}_{4}$ in $\mathrm{N}_{2}$ at 760 Torr (part of the $\mathrm{R}$ branch of the $2 v_{3}$ band) acquired with a stabilized spectrometer. (a) Spectrum from a single scan (black markers) compared to a fit of the Vernier model (red curve). (b) Residual of the fit.

The average noise on the baseline, estimated in a similar way as described in section 4.3 , is 3.5 $\times 10^{-3}$ in $50 \mathrm{~ms}$, which corresponds to NEAS of $1 \times 10^{-7} \mathrm{~cm}^{-1} \mathrm{~Hz}^{-1 / 2}$ (assuming $L_{\mathrm{eff}}=70 \mathrm{~m}$ ) and the figure of merit of $8 \times 10^{-9} \mathrm{~cm}^{-1} \mathrm{~Hz}^{-1 / 2}$ (assuming $M=2.7 \mathrm{THz} / 13 \mathrm{GHz}=207$ spectral elements). The lower NEAS compared to the $1576 \mathrm{~nm}$ range is mostly the consequence of the lower laser power and the lower finesse in this spectral region.

\section{Conclusions}

We demonstrated a new, improved design of a continuous-filtering Vernier spectrometer based on a compact fs Er-doped fiber laser and a rotating aperture that selects one Vernier order. The simplified and more robust spectrometer acquires spectra with similar resolution as in previous demonstrations of the technique (a few GHz), but at 5 times faster rates (up to $100 \mathrm{~Hz}$ ) [28,29,31]. 
Some spectra were acquired at scanning speeds above the adiabatic limit, and we introduced a simple Lorentzian correction function to the theoretical model of the absorption spectrum that well reproduces the reduction of the signal at these scanning speeds. The spectrometer allows recording the entire emission spectrum of the fs laser in one scan, and we demonstrate its operation around two center wavelengths $-1575 \mathrm{~nm}$ and $1650 \mathrm{~nm}$, where the latter is demonstrated for the first time using this technique. We record spectra of $\mathrm{CO}_{2}$ covering $1.7 \mathrm{THz}$ of bandwidth at 1575 $\mathrm{nm}$, and of $\mathrm{CH}_{4}$, covering $2.7 \mathrm{THz}$ at $1650 \mathrm{~nm}$. The noise equivalent absorption at $1575 \mathrm{~nm}$ is $5 \times 10^{-9} \mathrm{~cm}^{-1} \mathrm{~Hz}^{-1 / 2}$, which is better than in previous demonstrations of the technique in the same spectral region, using a spectrometer with the galvo scanner and the same Er-doped fiber laser [31] or a commercial comb source [29], or using a Czerny-Turner spectrometer with scanned mirror and a CCD camera [36]. Moreover, the demonstrated absorption sensitivity is comparable to that of other robust cavity-enhanced techniques, e.g. off-axis integrated cavity output spectroscopy [37], while the spectral bandwidth is significantly increased.

The spectrometer does not require a fast active feedback to the laser $f_{\text {rep }}$. Instead, only a slow feedback to the cavity length is needed for long-term operation of the spectrometer. This implies that the spectrometer can be used interchangeably with different kinds of lasers operating in the same spectral range, and does not require any fast actuators in the laser. Therefore this detection scheme is particularly attractive for the mid-infrared range, where the use of optical parametric oscillators [28] or difference frequency generation sources makes the fast feedback cumbersome to implement. The free-running capability of the spectrometer combined with the compactness of the laser source represents a significant step towards robust cavity-enhanced optical frequency comb spectroscopy, which can lead to field-deployable cavity-enhanced instruments.

Funding. Knut och Alice Wallenbergs Stiftelse (KAW 2015.0159); Fundacja na rzecz Nauki Polskiej (First TEAM/2017-4/39); Narodowa Agencja Wymiany Akademickiej (PPI/APM/2018/1/00031/U/001).

Disclosures. The authors declare no conflicts of interest.

Data availability. Data underlying the results presented in this paper are not publicly available at this time but may be obtained from the authors upon reasonable request.

\section{References}

1. D. Romanini, A. A. Kachanov, N. Sadeghi, and F. Stoeckel, "CW cavity ring down spectroscopy," Chem. Phys. Lett. 264(3-4), 316-322 (1997)

2. F. M. Schmidt, O. Vaittinen, M. Metsala, M. Lehto, C. Forsblom, P. H. Groop, and L. Halonen, "Ammonia in breath and emitted from skin,” J. Breath Res. 7(1), 017109 (2013).

3. D. S. Baer, J. B. Paul, J. B. Gupta, and A. O'Keefe, "Sensitive absorption measurements in the near-infrared region using off-axis integrated-cavity-output spectroscopy," Appl. Phys. B 75(2-3), 261-265 (2002).

4. D. S. Sayres, E. J. Moyer, T. F. Hanisco, J. M. St Clair, F. N. Keutsch, A. O’Brien, N. T. Allen, L. Lapson, J. N. Demusz, M. Rivero, T. Martin, M. Greenberg, C. Tuozzolo, G. S. Engel, J. H. Kroll, J. B. Paul, and J. G. Anderson, "A new cavity based absorption instrument for detection of water isotopologues in the upper troposphere and lower stratosphere,” Rev. Sci. Instrum. 80(4), 044102 (2009).

5. J. Morville, S. Kassi, M. Chenevier, and D. Romanini, "Fast, low-noise, mode-by-mode, cavity-enhanced absorption spectroscopy by diode-laser self-locking," Appl. Phys. B 80(8), 1027-1038 (2005).

6. L. Lechevallier, R. Grilli, E. Kerstel, D. Romanini, and J. Chappellaz, "Simultaneous detection of $\mathrm{C}_{2} \mathrm{H}_{6}, \mathrm{CH}_{4}$, and $\delta^{13} \mathrm{C}_{-} \mathrm{CH}_{4}$ using optical feedback cavity-enhanced absorption spectroscopy in the mid-infrared region: towards application for dissolved gas measurements," Atmos. Meas. Tech. 12(6), 3101-3109 (2019).

7. M. N. Fiddler, I. Begashaw, M. A. Mickens, M. S. Collingwood, Z. Assefa, and S. Bililign, "Laser spectroscopy for atmospheric and environmental sensing," Sensors 9(12), 10447-10512 (2009).

8. B. Henderson, A. Khodabakhsh, M. Metsala, I. Ventrillard, F. M. Schmidt, D. Romanini, G. A. D. Ritchie, S. T. Hekkert, R. Briot, T. Risby, N. Marczin, F. J. M. Harren, and S. M. Cristescu, "Laser spectroscopy for breath analysis: towards clinical implementation,” Appl. Phys. B 124(8), 161 (2018).

9. A. Foltynowicz, P. Maslowski, A. J. Fleisher, B. J. Bjork, and J. Ye, "Cavity-enhanced optical frequency comb spectroscopy in the mid-infrared - application to trace detection of hydrogen peroxide," Appl. Phys. B 110(2), $163-175$ (2013)

10. R. Grilli, G. Mejean, S. Kassi, I. Ventrillard, C. Abd Alrahman, E. Fasci, and D. Romanini, "Trace measurement of $\mathrm{BrO}$ at the ppt level by a transportable mode-locked frequency-doubled cavity-enhanced spectrometer," Appl. Phys. B 107(1), 205-212 (2012). 
11. R. Grilli, G. Mejean, S. Kassi, I. Ventrillard, C. Abd-Alrahman, and D. Romanini, "Frequency comb based spectrometer for in situ and real time measurements of $\mathrm{IO}, \mathrm{BrO}, \mathrm{NO}_{2}$, and $\mathrm{H}_{2} \mathrm{CO}$ at pptv and ppqv levels," Environ. Sci. Technol. 46(19), 10704-10710 (2012).

12. B. Bernhardt, A. Ozawa, P. Jacquet, M. Jacquey, Y. Kobayashi, T. Udem, R. Holzwarth, G. Guelachvili, T. W. Hansch, and N. Picque, "Cavity-enhanced dual-comb spectroscopy," Nat. Photonics 4(1), 55-57 (2010).

13. S. Kassi, K. Didriche, C. Lauzin, X. D. D. Vaernewijckb, A. Rizopoulos, and M. Herman, "Demonstration of cavity enhanced FTIR spectroscopy using a femtosecond laser absorption source," Spectroc. Acta A 75(1), 142-145 (2010).

14. A. Foltynowicz, T. Ban, P. Maslowski, F. Adler, and J. Ye, "Quantum-noise-limited optical frequency comb spectroscopy," Phys. Rev. Lett. 107(23), 233002 (2011).

15. P. Maslowski, K. F. Lee, A. C. Johansson, A. Khodabakhsh, G. Kowzan, L. Rutkowski, A. A. Mills, C. Mohr, J. Jiang, M. E. Fermann, and A. Foltynowicz, "Surpassing the path-limited resolution of Fourier-transform spectrometry with frequency combs," Phys. Rev. A 93(2), 021802 (2016).

16. A. J. Fleisher, D. A. Long, Z. D. Reed, J. T. Hodges, and D. F. Plusquellic, "Coherent cavity-enhanced dual-comb spectroscopy," Opt. Express 24(10), 10424-10434 (2016).

17. N. Hoghooghi, R. J. Wright, A. S. Makowiecki, W. C. Swann, E. M. Waxman, I. Coddington, and G. B. Rieker, "Broadband coherent cavity-enhanced dual-comb spectroscopy," Optica 6(1), 28-33 (2019).

18. W. P. Zhang, X. Y. Chen, X. J. Wu, Y. Li, and H. Y. Wei, "Adaptive cavity-enhanced dual-comb spectroscopy," Photon. Res. 7(8), 883-889 (2019).

19. M. J. Thorpe, D. Balslev-Clausen, M. S. Kirchner, and J. Ye, "Cavity-enhanced optical frequency comb spectroscopy: application to human breath analysis," Opt. Express 16(4), 2387-2397 (2008).

20. K. C. Cossel, F. Adler, K. A. Bertness, M. J. Thorpe, J. Feng, M. W. Raynor, and J. Ye, "Analysis of trace impurities in semiconductor gas via cavity-enhanced direct frequency comb spectroscopy,” Appl. Phys. B 100(4), 917-924 (2010).

21. G. Kowzan, K. F. Lee, M. Paradowska, M. Borkowski, P. Ablewski, S. Wojtewicz, K. Stec, D. Lisak, M. E. Fermann, R. S. Trawinski, and P. Maslowski, "Self-referenced, accurate and sensitive optical frequency comb spectroscopy with a virtually imaged phased array spectrometer," Opt. Lett. 41(5), 974-977 (2016).

22. C. Gohle, B. Stein, A. Schliesser, T. Udem, and T. W. Hansch, "Frequency comb Vernier spectroscopy for broadband, high-resolution, high-sensitivity absorption and dispersion spectra," Phys. Rev. Lett. 99(26), 263902 (2007).

23. L. Rutkowski and J. Morville, "Broadband cavity-enhanced molecular spectra from Vernier filtering of a complete frequency comb," Opt. Lett. 39(23), 6664-6667 (2014).

24. M. S. de Cumis, R. Eramo, N. Coluccelli, M. Cassinerio, G. Galzerano, P. Laporta, P. De Natale, and P. C. Pastor, "Tracing part-per-billion line shifts with direct-frequency-comb Vernier spectroscopy," Phys. Rev. A 91(1), 012505 (2015).

25. M. S. de Cumis, R. Eramo, N. Coluccelli, G. Galzerano, P. Laporta, and P. C. Pastor, "Multiplexed direct-frequencycomb Vernier spectroscopy of carbon dioxide $2 v_{1}+v_{3}$ ro-vibrational combination band," J. Chem. Phys. 148(11), 114303 (2018).

26. G. Kowzan, D. Charczun, A. Cygan, R. S. Trawinski, D. Lisak, and P. Maslowski, "Broadband optical cavity mode measurements at Hz-level precision with a comb-based VIPA spectrometer," Sci. Rep. 9(1), 8206 (2019).

27. L. Rutkowski and J. Morville, "Continuous Vernier filtering of an optical frequency comb for broadband cavityenhanced molecular spectroscopy,” J. Quant. Spectrosc. Radiat. Transf. 187, 204-214 (2017).

28. A. Khodabakhsh, L. Rutkowski, J. Morville, and A. Foltynowicz, "Mid-infrared continuous-filtering Vernier spectroscopy using a doubly resonant optical parametric oscillator,” Appl. Phys. B 123(7), 210 (2017).

29. C. Lu, F. Senna Vieira, F. M. Schmidt, and A. Foltynowicz, "Time-resolved continuous-filtering Vernier spectroscopy of $\mathrm{H}_{2} \mathrm{O}$ and $\mathrm{OH}$ radical in a flame," Opt. Express 27(21), 29521-29533 (2019).

30. D. Romanini, I. Ventrillard, G. Méjean, J. Morville, and E. Kerstel, "Introduction to cavity enhanced absorption spectroscopy", in G. Gagliardi and H.-P. Loock, Cavity-Enhanced Spectroscopy and Sensing (2014).

31. A. Głuszek, F. Senna Vieira, A. Hudzikowsi, A. Wąż, J. Sotor, A. Foltynowicz, and G. Sobon, "Compact mode-locked Er-doped fiber laser for broadband cavity-enhanced spectroscopy,” Appl. Phys. B 126(8), 137 (2020).

32. I. Silander, P. Ehlers, J. Y. Wang, and O. Axner, "Frequency modulation background signals from fiber-based electro optic modulators are caused by crosstalk," J. Opt. Soc. Am. B 29(5), 916-923 (2012).

33. I. E. Gordon, L. S. Rothman, C. Hill, R. V. Kochanov, Y. Tan, P. F. Bernath, M. Birk, V. Boudon, A. Campargue, K. V. Chance, B. J. Drouin, J. M. Flaud, R. R. Gamache, J. T. Hodges, D. Jacquemart, V. I. Perevalov, A. Perrin, K. P. Shine, M. A. H. Smith, J. Tennyson, G. C. Toon, H. Tran, V. G. Tyuterev, A. Barbe, A. G. Csaszar, V. M. Devi, T. Furtenbacher, J. J. Harrison, J. M. Hartmann, A. Jolly, T. J. Johnson, T. Karman, I. Kleiner, A. A. Kyuberis, J. Loos, O. M. Lyulin, S. T. Massie, S. N. Mikhailenko, N. Moazzen-Ahmadi, H. S. P. Muller, O. V. Naumenko, A. V. Nikitin, O. L. Polyansky, M. Rey, M. Rotger, S. W. Sharpe, K. Sung, E. Starikova, S. A. Tashkun, J. Vander Auwera, G. Wagner, J. Wilzewski, P. Wcislo, S. Yu, and E. J. Zak, "The HITRAN2016 molecular spectroscopic database," J. Quant. Spectrosc. Radiat. Transf. 203, 3-69 (2017).

34. P. Werle, R. Mucke, and F. Slemr, "The limits of signal averaging in atmospheric trace-gas monitoring by Tunable Diode-Laser Absorption Spectroscopy (TDLAS)," Appl. Phys. B 57(2), 131-139 (1993).

35. F. Adler, P. Maslowski, A. Foltynowicz, K. C. Cossel, T. C. Briles, I. Hartl, and J. Ye, "Mid-infrared Fourier transform spectroscopy with a broadband frequency comb," Opt. Express 18(21), 21861-21872 (2010). 


\section{Optics EXPRESS}

36. F. Zhu, J. Bounds, A. Bicer, J. Strohaber, A. A. Kolomenskii, C. Gohle, M. Amani, and H. A. Schuessler, "Near infrared frequency comb Vernier spectrometer for broadband trace gas detection," Opt. Express 22(19), 23026-23033 (2014).

37. J. J. Wang, X. Tian, Y. Dong, G. D. Zhu, J. J. Chen, T. Tan, K. Liu, W. D. Chen, and X. M. Gao, "Enhancing off-axis integrated cavity output spectroscopy (OA-ICOS) with radio frequency white noise for gas sensing," Opt. Express 27(21), 30517-30529 (2019). 